\title{
TEAM WORKING IN PSYCHIATRY
}

\author{
Meena Agarwal, MRCPsych, LLM
}

Potential workloads in Child and Adolescent Psychiatry have led to discussions within the professions about 'who does what' and attempts to redefine the role of doctors. Dr Meena Agarwal, consultant in child and adolescent psychiatry, describes how such a model for working might be translated into adult psychiatry practice. It has implications for all consultants.

\section{INTRODUCTION}

An estimated $10 \%$ of $5-15$ year olds have a recognisable mental health disorder. This means that approximately 1.1 million children and young people under 18 would benefit from specialist mental health services ${ }^{(1)}$. Some 45,000 young people have a severe mental health disorder and approximately $40 \%$ of children with a mental disorder do not currently have access to any specialist service. A similar number of children and young people with less serious mental problems may also need some help.

The National Service Framework (NSF) for Children ${ }^{(1)}$ has established clear standards for promoting the health and wellbeing of all children and young people and for providing high quality services to meet their needs.

One of the key factors to ensure successful delivery of the NSF is the workforce on whom we depend for delivering care and the services which children have a right to expect. The NSF recommends that the local commissioners ensure that their workforce is sufficient in size, skilled, well-led and well-supported to deliver the required high quality services for children and adolescents.

Implementing the standards of NSF requires service changes. These changes apply to the number and type of staff employed and the value of working in partnership. Services of the future will need a multiskilled workforce. The NSF has provided guidelines on developing high quality multidisciplinary child and adolescent mental health (CAMHS) teams.

Workforce planning is an extremely serious issue for CAMHS $^{(2)}$. Specialist CAMHS for children with mental health problems need to be properly resourced with highly trained professionals and practitioners. However, there is currently a huge shortage of such professionals and there is an urgent need to train sufficient numbers of staff to be able to deal with the complexity of children/adolescents with mental health problems. One way of addressing the shortage of professionals is to examine existing work practices and develop new ways of working.

Child mental health services are delivered by teams of multidisciplinary staff which, together, offer comprehensive care. No individual staff group is expected to possess all of the capabilities, knowledge and the skills required. There is a requirement of staff with a range of capabilities.
Some professional skills are unique to staff groups. For example, the Responsible Medical Officer's (RMO) responsibilities under the Mental Health Act (MHA) for psychiatrists are directed by law. Others are shared across several groups (eg Cognitive Behaviour Therapy), while generic skills, such as good communication, are common to the whole workforce.

Work has been progressing to develop a matrix to help determine the tasks of staff groups within the mental health workforce $^{(2)}$. Key questions addressed by the 2004 Cross Boundary Subgroup are:

- what are unique, shared and common tasks and responsibilities of individual staff groups?

- what tasks can be delegated to other groups under what conditions; if any?

- what tasks are not met by the existing workforce?

Following the introduction of the NSF standards for CAMHS three changes were suggested:

- increasing the workforce capacity and productivity through skill mix and continuing professional development

- moving work from doctors to other health care professionals

- moving work from health care professionals to the support workforce, supported by pay modernisation and service design

The review of roles, responsibilities and working practices across the role boundaries and between the professions has been essential because of the limited supply of staff in all professional groups. Consultations with the Royal College of Psychiatrists (RCPsych), Department of Health (DoH) and the British Medical Association (BMA) have led to an exploration of new ways of working for consultant psychiatrists and other professionals involved in delivering mental health care across the United Kingdom. The National Institute of Mental Health in England (NIMHE) ${ }^{(3)}$ and the RCPsych have reviewed the role of psychiatrists and their interface with other professionals providing mental health care. The National Steering Group (NSG) explored the professional role of psychiatrists in the context of multidisciplinary working ${ }^{(4)}$. Their aims included commissioning projects to identify challenges and solutions and test new ways of working.

This article attempts to summarise the findings of various national reports in relation to the new ways of working for consultant psychiatrists within the multidisciplinary CAMHS teams. As an illustration, references have been made to the local framework produced by the Avon and Wiltshire Mental Health Partnership Trust ${ }^{(5)}$. The aims of this article include the provision of information for the other Trusts so that they can consider how this framework might be used locally. 


\section{NEW ROLES FOR PSYCHIATRISTS}

The BMA published a report from the cross agency NSG on the New Roles for Psychiatrists in February 2004(6). The key areas of concern in relation to the role of the psychiatrists include:

- issues concerning responsibilities, power and accountability

- training in leadership and management skills

- concerns about ensuring continuity of care

The traditional role of consultants, not only in psychiatry but also in other fields of secondary care, has been that they carry responsibility for all patients under the care of the service. In psychiatry, the position is stated in law in respect of patients detained under the MHA, where this responsibility is clear. No such legal constraints exist to prevent full responsibility for other work being delegated to appropriately qualified nursing and allied health professionals, but, in practice, the mere existence of a law defining consultants' responsibility in one aspect of practice has hindered the development of expanding the scope of practice of others, for fear of legal repercussions. This results in unmanageable workloads for consultants in a shortage specialty.

Similarly, assumptions that consultant psychiatrists should automatically be team leaders have led to role confusion in some areas, to stress for consultants and to irritation from other professionals.

Many psychiatrists recognise that this situation is undesirable. The RCPsych Research Unit has measured the effect of different consultant roles on their level of stress ${ }^{(6)}$. It showed that an alarming proportion of consultants exceeded clinically significant scores on well-validated questionnaires. Consultants who adopted new ways of working were significantly less stressed as compared to those who practised more traditionally.

Research indicated that moving from the more traditional 'psychiatrist centred service' to a more 'consultative partnership' style could lead to jobs that reduce stress, burn out and actual working hours of psychiatrists and create more opportunities for other disciplines.

In such a style, psychiatrists operate as consultant advisers to a team or teams. When working in this way consultant psychiatrists advise or consult with the other professionals. In these instances, consultants are responsible for the quality and content of the advice they offer, but do not adopt continuing responsibility for the care of the service users. Service users' care is managed through care co-ordination and the other professionals retain clinical responsibility for their practice. This enables psychiatrists to have reduced caseloads concentrating on the care of people with high levels of severity and complexity of problems.

Success in using consultants in these more strategic ways requires clarification about accountability, supervision and leadership in mental health care. There is a need to review RCPsych guidance on job descriptions and recommended norms for numbers of psychiatrists in a service.

The roles and responsibilities of all staff groups will change as a consequence of psychiatrists adopting new roles. There is a need to develop further roles at advanced and consultant levels across many other professional groups and to design new roles at assistant and associate level to facilitate clinical career progression.

\section{RATIONALISATION OF CONSULTANT WORKLOAD} AND RESPONSIBILITY

Avon and Wiltshire Mental Health Partnership Trust has produced a local framework ${ }^{(5)}$ which has been agreed with its lawyers and Clinical Negligence Support for Trusts. It has been approved by its Trust Board. Key features of this Framework are summarised below, with its permission.

\section{The problem}

Confusion exists as to the limits of the role of the consultant psychiatrist. Many other professionals think that the legal responsibilities of consultant psychiatrists extend well beyond those defined by the Mental Health Act. This conflict risks the production of job descriptions for consultants that are not manageable and risks wasting skilled medical resources on work that could be delegated. In order to effect new ways of working for both consultants and their colleagues within the multidisciplinary team the limits of medical responsibility need to be clarified.

\section{The proposed solution}

Individuals, of whatever profession, are responsible for the quality of care that they provide to individual patients and accountable if it falls below acceptable standards. Team members will have a line manager, and a professional head, who may or may not be the line manager.

\section{It follows from this that:}

a) Doctors are not responsible for the quality of care provided by another team member.

b) There is no requirement to have the consultant's name on the notes of an outpatient who is not actually being seen by the consultant.

c) Consultants do not have to consider that their total caseload is the combined caseloads of all the other team members plus their personal caseload.

d) If the consultant does not see a patient, but provides advice, he/she is responsible for the quality of that advice, but not responsible for the ongoing care provided by others, or whether the advice is taken or not. 'Care co-ordination' is not a good use of consultants' time.

e) A designated clinical team leader takes responsibility for the organisation of processes within the team to ensure its smooth operation, eg systerns around referrals and allocation. This person needs to be a senior clinician within the team, but could come from any profession. Referrers should be made aware that this person is to be contacted with any queries relating to the referral process itself.

The consultant workload

- triaging of referrals by a senior clinician, especially in areas of medical staff shortages

- multidisciplinary assessments

- improved caseload managements with teams, a 'stepped care' approach to the provision of secondary care

- paving the way for crisis team, gate-keeping of inpatient beds and facilitating early discharge

\section{Practicalities}

Teams needs to have robust processes for the allocation of work, management of risk and integrated care planning. If resources are insufficient to meet demand this needs to be recorded and the reasons for not providing care given. In the case of a legal challenge, it is often the processes which get challenged rather than the actual clinical decisions.

\section{CONSULTANT RESPONSIBILITIES IN TODAY'S NHS}

The RCPsych sought the views on consultant psychiatrists responsibilities in late $2003^{(6)}$. These views were sought on three hypothetical illustrative options for new roles for psychiatrists and other mental health professionals and on how work, roles and responsibilities might be distributed between them. 
These options are:

\section{Option 1}

No change in consultant psychiatrists' responsibilities.

The main features of this option are that:

- consultants continue to be responsible for very large numbers of patients in secondary care

- consultants accept continuing workload problems and the likelihood of further increases in demand

- consultants hope that more colleagues will be recruited from the increased output of medical schools - this is likely to require us to wait a decade or more for any significant impact on consultant numbers

- there is a much lesser requirement for professionals from other disciplines to develop greater clinical autonomy

\section{Option 2}

Smaller and selected consultant caseloads with responsibility for other patients delegated to other disciplines in the teams that provide services.

The main features of this option are that:

- consultants have reduced personal caseloads, but continue to be responsible for very large numbers of patients

- there is a named consultant for all patients whether they are inpatients or outpatients in secondary care

- consultants develop supervisory relationships with other professionals

- the other disciplines need to develop competencies so that they can accept greater clinical autonomy

- there is no need to negotiate with the General Medical Council (GMC) with the aim of developing its current guidance

- there would be the need to develop guidelines to define the meaning, nature and range of 'complexity' and the training required to address it

\section{Option 3}

Smaller and selected consultant caseloads with responsibility for other patients distributed among other professionals in teams.

The main features of this option are that:

- consultants have reduced caseloads and direct responsibility for fewer patients in secondary care

- consultants have clinical responsibility for selected high risk or complex cases

- consultants develop consultancy relationships with other professionals

- professionals from other professions need to develop competencies for clinical autonomy

- there may be a need for the RCPsych to request the GMC to develop guidance on the responsibilities of consultant psychiatrists

- there would be the need to develop guidelines to define the meaning, nature and range of 'complexity' and the training required to address it

The responses indicated that Option 1 (no change) was not popular. The majority favoured Option 3 and a sizeable minority saw Option 2 as more easily attainable or preferable.
The Avon and Wiltshire Mental Health Partnership Trust guidance was originally produced for the consultants working in adult psychiatry. No formal guidance exists that may apply specifically to the consultants working in child and adolescent psychiatry. However, the guidance was discussed in a workshop entitled 'Psychiatry in CAMHS' at the CAMHS National Conference on understanding workforce issues in $2004^{(6)}$. The general view was that this guidance can be usefully applied to the consultant child and adolescent psychiatrist working in multidisciplinary teams.

Both the 'Psychiatry in CAMHS' workshop discussions and the informal discussions with regional consultant child and adolescent psychiatrists suggest that a variety of working models exist nationwide, including the north west region. It appears that the preferred way of service delivery in most CAMHS services in the north west, with the exception of a few, has been similar to what has been described in Option 1 above. However, it seems that this model of service delivery may not be viable in the long term due to the shortage of consultant child and adolescent psychiatrists nationwide. From informal discussions with consultant colleagues, it is my impression that, like the general adult psychiatric colleagues, a service model based on Option 3 as above would be the most preferred way of working in today's NHS and in the future.

\section{CONCLUSION}

This article has summarised only some of the key points relevant to this topic. The legal and professional responsibilities of consultant psychiatrists are still being actively discussed by the RCPsych, the BMA and the GMC to provide greater clarity and flexibility nationally. Capable organisations should rely on good systems, structures and characteristics which affect the functioning of staff.

\section{ACKNOWLEDGEMENT}

Avon and Wiltshire Mental Health Partnership Trust for permission to republish, in parts, their Trust Guidance on the role of consultant psychiatrists.

\section{REFERENCES}

1. National Service Framework for Children, Young People and Maternity Services. The Mental Health and Psychological Wellbeing of Children and Young People. London: DH Publications; 2004

2. Nixon B. Towards an Understanding of Workforce Issues in CAMHS. IN CAMHS National Conference, Understanding Workforce Issues in CAMHS Conference Handbook. Department of Education and Skills, Department of Health; 2004

3. NIMHE. The Ten Essential Shared Capabilities: A Framework for the Whole of the Mental Health Workforce. London: DH Publications; 2004

4. Department of Health, National Steering Group, Guidance on New Ways of Working for Psychiatrists in a Multidisciplinary and Multi-agency Context - Interim Report. August 2004

5. Trust Guidance on the Role of the Consultant Psychiatrist. Avon and Wiltshire Mental Health Partnership NHS Trust. 26 September 2003

6. Department for Education and Skills, Department of Health. CAMHS National Conference. Understanding Workforce Issues in CAMHS. Birmingham: 29 November 2004 